\title{
La réutilisation du patrimoine défensif urbain en Algérie
}

\author{
Reuse of urban defensive heritage in Algeria
}

\author{
Amina Korichi ${ }^{\text {a }}$, Zineeddine Guenadez ${ }^{b}$, Nicolas Faucherre ${ }^{c}$ \\ ${ }^{a}$ Université Mouloud Mammeri, Tizi Ouzou, Algeria, korichi.pg2009@yahoo.fr

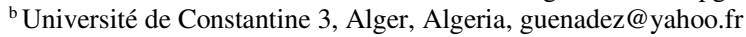 \\ c Laboratoire d'Archéologie Médiévale et Moderne en Méditerranée - Université d'Aix-Marseilles, France, \\ faucherrenicolas@gmail.com
}

\begin{abstract}
The growing interest in the heritage that contemporary society carries with it, and which is reflected in the extent of the debates and issues relating to its preservation and enhancement, runs up, most of the time, to the authentic memory / memory dichotomy. Dynamic. That being said, the reuse of built heritage in the process of renewing the image of our cities (use-value) becomes dependent on a global conception included in a sustainable urban development approach.

The monumental heritage, subdivided, generally, into five categories namely; religious, hospitable and funeral, civil (public or private), agricultural and industrial and finally military, raises enormous problems as for its reuse. These are more pronounced towards the military-defensive architecture that is the subject of this contribution.

In Algeria, defensive architecture occupies an important part in its heritage site. The typological diversity -the result of a long stratification from the early antiquity to the middle of the last century- as well as the dominant position occupied by this defensive heritage abandoned in the current urban landscape imposes a more urgent care.

Therefore, we want, through this communication, to lay the first steps of a patrimonial approach to highlight this or these unknown military architectures and preserve our defensive heritage for which the collective memory is not attested and still painful.

The reuse of the defensive heritage in Algeria must first and foremost go through its identification, inventory its various typologies, evaluate its current physical impact and finally explore the different urban alternatives that will result from its reintegration into the urban experience and for sustainable cities and citizens.
\end{abstract}

Keywords: Reuse, defensive heritage, urban, Algeria.

\section{Introduction}

Le paysage urbain que nous offrent les villes algériennes semble à priori, être le résultat d'une évolution faite de juxtaposition et superposition de plusieurs cultures urbaines relatives aux forces dominantes à un moment ou un autre des lieux. Les carthaginois, les romains, les vandales, les dynasties berbéro-musulmanes (Ham- madides, Almohades et Hafsides), les espagnols, les ottomans puis les français ont marqué l'espace urbain. Cette emprunte est extrêmement lisible sur le système défensif qui orne la structure urbaine de nos villes.

Aujourd'hui ce legs riche en formes et traces, est malheureusement non reconnu, abandonné et 
mal réutilisé. D'ailleurs mis à part quelques citadelles, remparts et portes des périodes musulmanes qui ont pu être consacrés monuments historiques durant le siècle dernier $\left(\mathrm{XX}^{\mathrm{ème}}\right)$, la patrimonialisation des autres productions espagnoles et françaises reste encore à attester.

Par cette communication, nous voulons mettre en relief l'intérêt patrimonial de la conservation de ce legs et sa consécration en monument historique.

\section{L'inventaire}

Nous avons dans le cadre de notre magister essayé de faire l'inventaire du patrimoine fortifié et ceci afin de dresser une sorte d'état des lieux de cette catégorie de monuments en Algérie. Il est à noter qu'il n'existe aucune liste recensant ce type de patrimoine dans notre pays. Cet essai d'inventaire non exhaustif est élaboré en consultant plusieurs documents et en contactant plusieurs organismes.

De manière plus générale, sur l'ensemble du parc classé algérien, 9.95\% sont des monuments fortifiés. Ces monuments classés sont d'époques différentes (romaine, byzantine, espagnole, arabo-musulmane et ottomane), à l'exception du colonial français qui ne trouve pas encore sa place dans ce parc.

Le nombre total des édifices que nous avons recensés sur l'ensemble du territoire est de 221 édifices et ceci en intégrant la période française.

\section{Répartition des monuments fortifiés}

Sur les 221 monuments inventoriés, 136 sont de l'époque coloniale soit plus de $61 \%$.

Le système défensif, complexe dans ses formes et fonctions, va offrir des espaces différents dont nous avons identifié les typologies principales.

\subsection{Les forts}

Ils représentent la typologie la plus dominante, plus de $67 \%$ des monuments inventoriés sont des forts militaires détachés.

Sur les $67 \%$ plus de $58 \%$ concerne l'héritage colonial. Ces forts militaires, en tant qu'entité, sont implantés plus dans la région sud de l'Algérie.

Cependant sur l'ensemble des forts inventoriés, $7,69 \%$ sont classés, dont $80 \%$ sont restaurés ou en cours de restauration.
Ne pouvant plus poursuivre leur fonction primaire liée à la défense, leur réutilisation se traduit à travers la fonction muséale qui est la plus dominante.

\subsection{Les portes}

Elles sont d'époques différentes (romaines, byzantines, médiévales, et françaises) et représentent $14 \%$ de l'ensemble des monuments inventoriés, dont $40 \%$ sont classées. Mais sur les $40 \%$, seul $27 \%$ sont restaurées ou en cours de restauration. Ces portes sont intégrées dans le paysage urbain sans pour autant bénéficier d'une réelle mise en valeur.

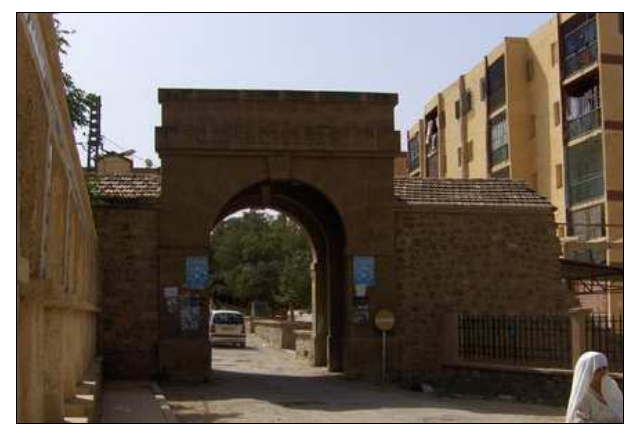

Fig. 1. Porte de Sour El Ghozlane.

\subsection{Les citadelles}

Pour un certain nombre d'entre elles, il s'agit de restes archéologiques. Elles bénéficient d'une protection sous forme de classement. Plusieurs projets de restauration de cette typologie sont lancés, mais jusqu'à ce jour aucun n'est finalisé comme le cas de la citadelle d'Alger et d'Annaba (Fig. 2).

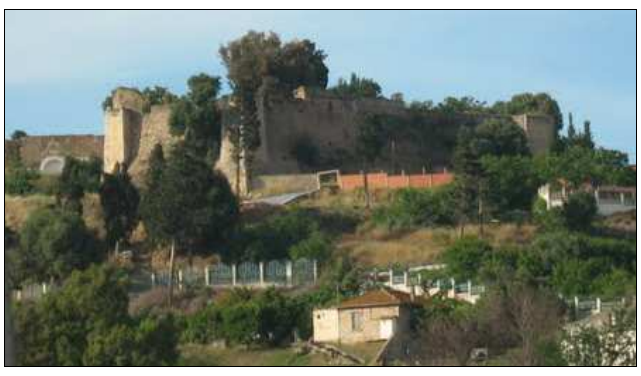

Fig. 2. La citadelle d'Annaba. 


\subsection{Les remparts}

Ils sont généralement des restes des anciennes structures, éparpillés dans des endroits isolés urbains ou dans des sites naturels. Dix sur dix-sept sites sont classés, dont un petit nombre de remparts qui est restauré comme celui de l'ancienne Tévhesse et ceux de Tlemcen (Fig. 3).

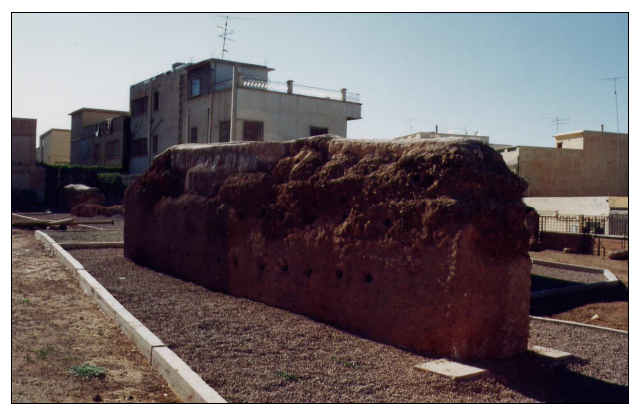

Fig. 3. Reste des remparts. Tlemcen.

\subsection{Les blockhaus, les batteries et les tours détachées}

C'est la typologie la plus négligée en matière de sauvegarde, bien qu'elle fasse partie du système défensif. On peut compter quelques restaurations comme celle de djebel Mestiri à Tébessa, classé récemment ou aussi bordj Ezafrani au niveau de Bab El Hadid à Tlemcen (Fig. 4).

\section{Le classement}

C'est tout au long du $\mathrm{XX}^{\text {ème }}$ siècle que la reconnaissance du patrimoine défensif a été initiée par l'autorité française qui a eu recours au classement comme outil de protection. Les démolitions de ce patrimoine ont été nombreuses dès l'occupation française puis dans les années 1900 où la plupart des villes du nord algérien

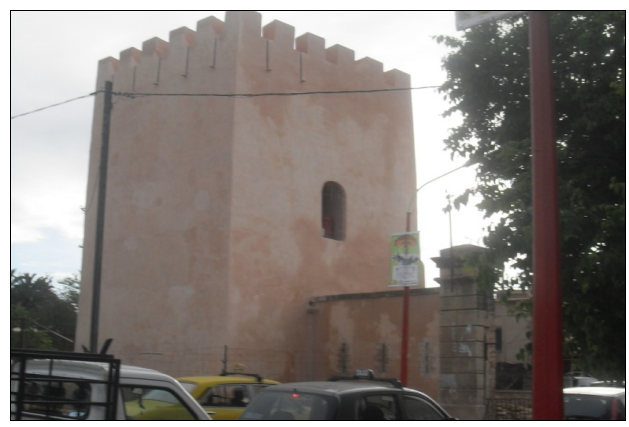

Fig. 4. Bordj Ezafrani. Tlemcen. vont connaitre un élargissement de leur périmètre urbain.

C'est aussi durant cette dernière période qu'il y a eu le plus de classement, qui s'est étalé de 1900 à 1912. La moitié des monuments défensifs y ont été classés Un arrêt de classement a été provoqué par la première guerre mondiale, il ne sera repris qu'après la guerre de la délibération nationale. Entre 1954 et 1962 le seul monument défensif qui a été classé est le rempart de Béni Isguen en 1956.

Après l'indépendance, quelques forts et remparts ont fait l'objet d'un classement mais la plupart d'entre eux sont d'époque romaine, byzantine ou arabo musulmane.

\section{La patrimonialisation du legs défensif colo- nial}

S'interroger sur le devenir des systèmes défensifs nécessite également de s'intéresser à leur patrimonialisation. "Le processus de patrimonialisation intervient souvent lorsqu'un lieu perd les valeurs pour lesquelles il a été créé et qu'il est conservé pour entretenir la mémoire, le témoignage, les vestiges du temps passé et pour lutter finalement contre l'oubli" (Gillot, 2008). La patrimonialisation des systèmes défensifs est un axe de réflexion important, qui interroge à la fois leur reconversion et leur intégration dans le vécu actuel. A l'inverse des autres productions architecturales, les systèmes défensifs peuvent provoquer une non-appropriation, voire un rejet, par la société vue leur histoire traumatisante. De ce fait, en Algérie, la consécration de cet héritage reste limitée à quelques citadelles, remparts et portes des périodes musulmanes, la patrimonialisation des autres productions espagnoles et françaises reste encore à attester (Korichi, 2011).

"Au moment où se développe dans le monde occidental une "inflation patrimoine" intégrant les produits du XXe siècle, l'Algérie, la Tunisie et le Maroc ne sont pas encore suffisamment conscients de la nécessité de promouvoir une politique destinée à l'héritage colonial" (Lazhar Gharbi, 2012). La question de la mise en valeur des systèmes défensifs coloniaux est quasiment occultée, bien que cette frange représente $62 \%$ de l'héritage militaire (Korichi, 2011). Certains auteurs stipulent que c'est le mouvement des générations qui refoule la mémoire des témoins directs et qui permet aux ouvrages défensifs leur consécration monument historique et leur appro- 
priation par le biais de leur réutilisation. D'autres voient, cet héritage comme "étant le produit d'un contact entre le Maghreb et l'Europe, le patrimoine colonial devrait être conçu comme l'expression de cette universalité des sociétés maghrébine" (Lazhar Gharbi, 2012). De ce fait sa patrimonialisation est acquise.

Le patrimoine défensif de l'époque colonial ou antérieur, est aujourd'hui abandonné, soumis aux aléas du temps et de l'homme, attestée par l'état de ruine de plusieurs monuments. Cet héritage est victime d'une rupture avec le passé douloureux, d'un problème identitaire de la société, mais il mérite d'être recensé, reconnue, classé et conservé.

\section{La réutilisation du patrimoine défensif en Algérie}

En raison de leur situation stratégique, et l'emprise foncière importante, les systèmes défensifs ont joué un rôle considérable dans l'implantation et l'aménagement des villes. Aujourd'hui, ces héritages militaires sis au cœur des villes ou sur des sites qui les dominent, mis à la retraite, ne retrouvent pas leur place dans la vie des citoyens. Le passage de la fonction défensive à la composante urbaine pose la question de leur intégration dans la dynamique du développement.

Le patrimoine défensif présente certaines spécificités liées à sa nature et à son rôle qui ont mené vers des formes et des espaces pas toujours faciles pour de nouveaux réaménagements ou des projets de reconversion. C'est également un patrimoine puissant accompagné par des exigences d'intendance.

En Algérie les monuments défensifs classés sont traités tout comme les autres monuments du parc patrimonial, sans aucune spécificité pour cette architecture militaire. La restauration et la réutilisation de ces monuments se fait sans prendre en compte leur particularité architecturale, et leur fonction d'origine. L'analyse de quelques projets de restauration / réutilisation de cette architecture montre très bien que cette indifférence engendre toutes sortes de mutilations et de dégradations irréversibles à notre patrimoine militaire.

Par ailleurs, sur le plan législatif, les textes relatifs à la protection du patrimoine ne précisent pas les formes de réutilisation de ces monuments. Ce vide législatif sur la manière d'intégrer les édifices défensifs dans la ville ou leur environnement ainsi que le manque d'outils théoriques induisent la muséification de ces monuments et leur isolement de leur contexte d'appartenance, ce qui accélère de la sorte leur dégradation.

La réutilisation de quelques monuments défensifs restaurés en Algérie se fait après l'opération de restauration où le choix de la fonction ne vient qu'en dernier ou sinon par besoin de l'heure. Cette rupture dans le processus de sauvegarde/réutilisation provoque parfois des dommages irréversibles aux monuments.

\section{Conclusion}

Nous avons pu à travers cette présentation démontrer que ce type de patrimoine, est très riche et varié à travers tout le territoire national. Le patrimoine défensif en Algérie est présent, mais d'une manière discrète et fragmentée. La majorité des villes algériennes ont perdu leur enceinte, ce qui reste du système défensif se résume à quelques fragments des murailles, des forts détachés, des casernes, des tours, et quelques portes.

L'inventaire nous a permis de montrer très bien que les édifices militaires coloniaux ne sont pas reconnus en tant que patrimoine national, malgré leur qualité architecturale et la multiplicité de leurs valeurs. Sur les 221 monuments recensés, 136 sont de l'époque coloniale, et aucun d'eux n'est classé. Ce problème de non reconnaissance de l'héritage colonial accélère la dégradation et la disparition de nombreux monuments défensifs tels que les forts, les remparts, et les portes.

La politique suivie par l'Algérie pour la sauvegarde de son patrimoine reste limitée à quelques mesures de protection telle que le classement au titre de monuments historiques. Leur mise en valeur de manière générale et par la réutilisation de manière spécifique, est absente ou minime, accentuée par les textes législatifs qui ne portent pas une attention particulière à ce type de patrimoine. 


\section{Bibliography}

Bourouiba, R. (1983). L'architecture militaire de l'Algérie médiévale, office des publications universitaires, l'Algérie, pp. 73-118.

Delerive, R. (1992). Forts sahariens des territoires du sud, éditions Geuthner, pp 5-120.

Gillot, L. (2008). "Archéologie et processus de patrimonialisation au Moyen-Orient : la mise en valeur du site archéologique de Bosra en Syrie", in Rencontres scientifiques en ligne de la Maison de l'Orient et de la Méditerranée, Lyon, p. 14.

Gsell, S. (1901). Les monuments antiques de l'Algérie, Albert Fontemoing éditeur, tom. I et II, pp. 10-250.

Khelifa, A. (2007). Histoire d'El Djazair, éditions Dalimen, pp. 170-204.

Korichi, A. (2011). La sauvegarde et la réutilisation des monuments du système défensif de la ville de Bejaia, Mémoire de magister, Université Mouloud Mammeri de Tizi-Ouzou, Faculté du Génie de la Construction, Département d’Arquitecture, pp. 51-87.

Lazhar Gharbi, M. (2012). "Le patrimoine colonial au Maghreb, disponible sur, p.2, in http://www.hermes.jussieu.fr (15-09-2012)

Marcais, G.; Marçais, W. (1903). Monuments arabes de Tlemcen, éditions tours, p. 118.

Ministère de la guerre. (1842-1843). Tableau de la situation des établissements français dans l'Algérie, imprimerie impériale, Paris, pp. 60-220.

Prelorenzo, C. (2011). "Patrimonialiser les bases de sous-marins et le Mur de l'Atlantique", In Situ, p. 3, in http://insitu.revues.org/312. 
